\title{
A Hematologist's Perspective of Performing Bone Marrow Aspiration and Biopsy by Interventional Radiologists
}

\author{
Anwarul Islam* \\ Division of Hematology/Oncology, Department of Medicine, Buffalo General Hospital, Buffalo, New York, USA
}

*Corresponding author: Anwarul Islam, Clinical Associate Professor of Medicine, Division of Hematology/Oncology, Department of Medicine, Buffalo General Hospital, Room E 318, Buffalo, New York 14203, USA

\begin{tabular}{|c|c|}
\hline ARTICLE INFO & ABSTRACT \\
\hline 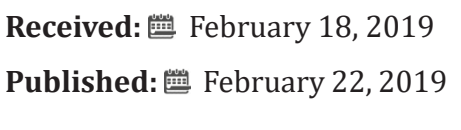 & $\begin{array}{l}\text { Citation: Anwarul Islam. A Hematologist's Perspective of Performing Bone Marrow As- } \\
\text { piration and Biopsy by Interventional Radiologists. Biomed J Sci \& Tech Res 15(1)-2019. } \\
\text { BJSTR. MS.ID.002634. }\end{array}$ \\
\hline
\end{tabular}

\section{Introduction}

Aspiration of hematopoietic bone marrow and the process of obtaining solid intact bone marrow core biopsy specimens are specialized medical procedures. They require a defined body of knowledge coupled with practiced surgical skill [1]. Until some years ago these specialized procedures were almost exclusively performed by hematologists and oncologists. In more recent years interventional radiologists have entered the field of hematopoietic bone marrow acquisition [2-6]. This trend has occurred for multiple reasons, particularly due to the desire to enhance the efficiency of the physician's activities, utilization of conscious sedation [7-9], the availability of suitable medical facilities, and the need for improved patient accommodation. Interventional radiologists are accustomed to performing one-step punch biopsies from the liver, kidney, lymph nodes and lung. These procedures do not require bedside preparation of slides from the obtained specimens. Bone marrow examination, on the other hand, requires a two-step procedure; one - for bone marrow aspiration (liquid marrow) and its immediate preparation of dry film smears and second - extracting a solid core (trephine) biopsy specimen.

Interventional radiologists are often ideal practitioners for accessing samples of hematopoietic marrow particularly in cases where the patient is very obese (Figure 1). In the latter circumstance, hematologists and oncologists are often not equipped to be able to perform the procedure with their customary efficiency. There are also concerns of safety and convenience for the patient. Conversely, it is beneficial for everyone involved in the procedure if the radiologist has some training in ensuring that good quality bone marrow specimens are derived for hematologic evaluation. This viewpoint is concerned with the interventional radiologist's endeavors in this area of medical practice. The interventional radiologist's function in bone marrow-related procedures is to obtain adequate samples in excellent condition ensuring a successful diagnostic evaluation can be carried out by the hematopathologist. The role of the interventional radiologist is to support and sustain requirements of the hematologists and oncologists who are responsible for the medical and hemato-oncologic care of the patient. It is also understood the interventional radiologist may be in a better position to obtain hematological samples from certain patients than the hematologist/oncologist who otherwise personally performs his/her bone marrow aspirations and biopsies.

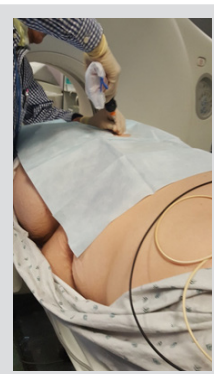

Figure 1: Shows a markedly obese woman undergoing bone marrow biopsy procedure by an interventional radiologist using an On-Control needle. 
One specific example is the extremely obese patient in whom routine aspiration and biopsy techniques are inadequate to access the bone marrow which is deeply placed and difficult to locate. In such circumstances a CT guidance [2-6] can play a crucial role in locating the biopsy site and procuring an adequate bone marrow sample. One common imperfection of the interventional radiology technique is to sequentially use the same bone marrow needle for both aspiration and biopsy. This is not an ideal practice [10]. Bone marrow aspiration before bone marrow core/trephine biopsy using the same bone marrow biopsy needle cause disruption of marrow tissue and results in biopsy specimens depleted or even denuded of bone marrow cells from the intertrabecular marrow spaces. Such artefactual change and tissue distortion interfere with proper histological evaluation and interpretation of core biopsy specimens. Another problem with their sampling methodology is that the radiologists typically do not make immediate bone marrow dry film smears. Their smears are usually prepared in the laboratory from anticoagulated samples that may await smearing for variable periods of time.

Since the morphology of the hematopoietic cells can deteriorate while standing in the anticoagulant, bone marrow smears should be made and air dried immediately after the aspiration is obtained. Additionally, alterations in cellular morphology due to delay in smear preparation versus those caused by some diseases can be difficult to differentiate. Bone marrow smears yield the best information when they are made from the initial concentrated marrow [i.e. the first small amount $(0.5 \mathrm{ml})$ of aspirated sample known as the first pull] [11]. If and when a large sample of marrow is aspirated in one pull with a $10 \mathrm{ml}$ or larger syringe (Figure 2) and placed in an anticoagulant, bone marrow smears derived from such pools of marrow after they have been sitting for a long period of time (sometimes hours by the time the specimen reaches the laboratory), yields smears that present diluted marrow with deteriorated cellular morphology and less than ideal numbers of hematopoietic cells. As emphasized earlier, the ideal scenario is for bone marrow smear preparation to be carried out immediately or as soon as possible from the first pull (about $0.5 \mathrm{ml}$ ) of marrow.

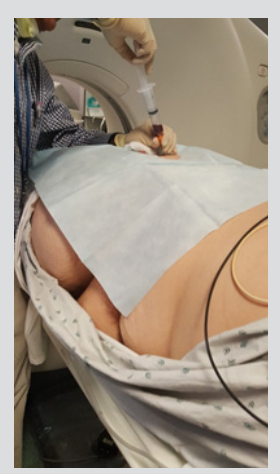

Figure 2: Shows bone marrow aspiration by an interventional radiologist using a $10 \mathrm{ml}$ syringe using the same On Control bone marrow biopsy needle.
The first initial pull $(0.5 \mathrm{ml})$ of marrow with a five-milliliter syringe should be immediately dispensed into a lavender (EDTA) tube for later use (shortly after the procedure is completed) for the preparation bone marrow smears. Additional marrow as required for other tests can be drawn with a $10 \mathrm{ml}$ or larger syringe and dispensed to appropriate collection tubes. Interventional radiologists typically withdraw $10 \mathrm{ml}$ or more marrow in one pull (Figure 2); as a result, the marrow is heavily diluted with blood and, contains a fewer number of spicules (marrow particles). Slides prepared from such specimens are inadequate for proper evaluation of the marrow particularly with regard to bone marrow cellularity. The intention of this submission is to help interventional radiologists from a hematologist's perspective to ensure that the procedures are performed effectively, the samples are collected in a proper manner, and placed in appropriate containers for

i. Morphology (lavender top tube/EDTA),

ii. Flow cytometry (green top tube/sodium heparin),

iii. Cytogenetics (green top tube/sodium heparin) and

iv. Molecular studies such as SNP array, FLT-3, IDH-1, IDH2, NPM1, BCR-ABL, JAK2, MPL mutation, etc. (green top tube/ sodium heparin).

These studies are now almost routinely performed for most patients undergoing bone marrow examination for hematological diagnosis, particularly in cases with acute and chronic myeloid leukemia and chronic myeloproliferative disorders. The posterior ilium tends to be the most used site for bone marrow aspiration and biopsy since it is the largest body of marrow near the body surface and it has been shown to be safer and more convenient to access. The optimal clinical evaluation of the bone marrow requires an examination of air dried and well stained smears of the aspirated tissue along with a histopathological evaluation of adequately processed core biopsy specimens. A bone marrow evaluation is essential in establishing a diagnosis, determining the efficacy of treatment in hematological disorders and monitoring hematologic status of patients following chemotherapy and bone marrow/stem cell transplantation. It is also an essential component of the staging process for newly diagnosed malignancies. Currently available bone marrow aspiration needles are quite satisfactory and if properly used provide good quality specimens for morphologic evaluation.

However, when a bone marrow core biopsy is concerned there are now several bone marrow biopsy needles that are currently in use and not all of them provide good quality biopsy specimens for histological evaluation nor are, they user friendly. We have compared the recently introduced Moeller Medical single-use bone marrow core biopsy needle with the Jamshidi needle with marrow acquisition cradle (CareFusion), J-needle (Cardinal Health) and On-Control device (Vidacare). It was concluded that the Moeller Medical needle system has definite advantages over the others and 
was recommended for routine use [12]. Interventional radiologists tend to prefer the On-Control device since it is power-driven, require decrease procedure time, reduces pain and yields improved biopsy specimens [13-15]. However, some of these favourable attributes to the On-Control needle are debatable [12]. One of the most important drawbacks of the On-Control needle system is that it has a helically distributed elevated ridge (a thread) on the terminal luminal surface of the needle (Figure 3). The purpose/ rationale of this thread is to capture and hold the biopsy specimen from slipping out of the needle as the needle is withdrawn from the patient's body.

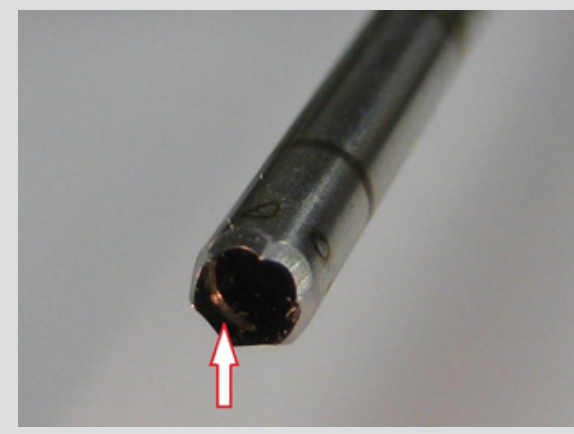

Figure 3: Shows the helically distributed elevated ridge (a thread) on the terminal luminal surface of the needle (arrow)

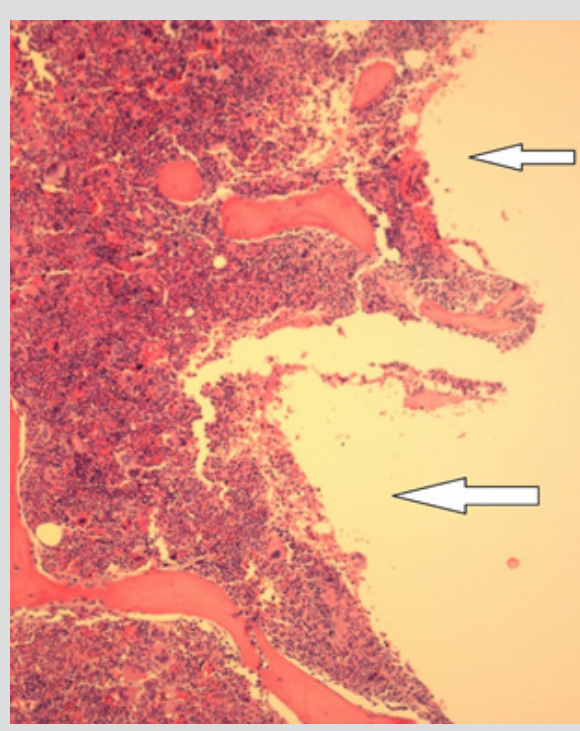

Figure 4: A histological section of a bone marrow biopsy specimen obtained with the On-Control needle. Note the groove (arrows) produced due to loss and removal of marrow tissue from the edge of the biopsy specimen caused by the rotating elevated internal ridge as it passes over the biopsy specimen during the biopsy procedure.

However, because of the presence of this internal thread near the tip of the needle that projects into its lumen and the high cutting speed of the drill, considerable damage is caused to the lateral edges of the biopsy specimen (Figure 4). The outcome is that the total width of the biopsy specimen is reduced for histological evaluation. Unlike the Moeller needle, the On-Control needle has the tendency to disrupt the longitudinal continuity of the biopsy specimen (Figure 5). Furthermore, it is difficult to eject the biopsy specimens from within the lumen of the needle as it is tightly held by the internal thread (Figure 6) near the distal cutting tip of the needle. As a result, excessive force is needed to remove the biopsy specimen with the ejector rod, and this may also cause compression artifact to the biopsy specimen. In recent years the specialty of interventional radiology has opened its specialized technique to the acquisition of aspiration and solid core biopsies of hematopoietic bone marrow. It is expected that this innovative and adaptive practice needs to be orientated to satisfy the morphologic, cytologic, and tissue requirements of contemporary diagnostic hematology. This viewpoint is written to identify and help remedy the undesired results when the radiologist's and the hematologist's diagnostic and clinical needs fail to seamlessly interdigitate. It is the intention for both the interventional radiologist' and the hematologist' to serve the best interest of any given patient. This is accomplished when the benefits and limitations of each specialty in this area of medicine are understood by all parties involved.

Figure 5: A schematic representation of a biopsy specimen obtained by an OnControl needle demonstrating disruption in the longitudinal continuity of the biopsy specimen.

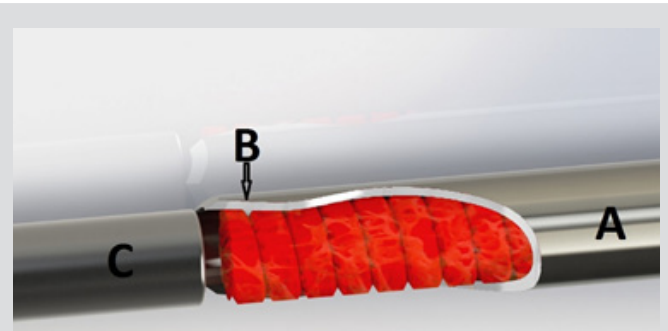

Figure 6: A schematic representation of a biopsy specimen obtained by an On Control needle.

I. Demonstrating difficulty in ejecting the biopsy specimen from within the lumen of the needle (A) because it is tightly held by the internally projected elevated ridge (B) near the distal cutting tip of the needle.

II. As a result excessive force is needed to remove the biopsy specimen with the ejector rod (C) which may cause compression artifact to the biopsy specimen. 


\section{References}

1. Islam A (2013) Manual of Bone Marrow Examination. ( $\left.2^{\text {nd }} E d n.\right)$, Trafford Publishers, USA, pp. 192.

2. Badiola CM, Scappaticci F, Brahaj D (2012) CT Guided Bone Marrow Aspiration and Core Biopsy. 2: 55-56.

3. Lack W, Donigan JA, Morcuende J, Buckwalter J, El-Khoury GY (2010) Conical utility of CT-guided biopsies in orthopaedic oncology. Iowa Orthop J 30: 76-79.

4. Le HBQ Lee ST, Munk PL (2010) Image-Guided Musculoskeletal Biopsies. Semin Intervent Radiol 27: 191-198.

5. Vadlamudi V, Sterling K (2017) Fluoroscopically guided powered bone marrow biopsy in interventional radiology: a safe and efficacious procedure. 28(2): S204.

6. Widlus DM, Moeslein FM, Iliescu B, Haskal ZJ (2009) An Approach to Routine Bone Marrow Aspiration and Biopsy (BMAB) by the Interventional Radiologist. 20(2): S137.

7. Mainwaring CJ, Wong C, Lush RJ, Smith JG, Singer CR (1996) The role of midazolam-induced sedation in bone marrow aspiration/trephine biopsies. Clin Lab Haematol 18(4): 285-288.

8. Giannoutsos I, Grech H, Maboreke T, Morgenstern G (2004) Performing bone marrow biopsies with or without sedation: a comparison. Clin Lab Haematol 26: 201-204.

\section{ISSN: 2574-1241}

DOI: 10.26717/BJSTR.2019.15.002634

Anwarul Islam. Biomed J Sci \& Tech Res

This work is licensed under Creative

Commons Attribution 4.0 License

Submission Link: https://biomedres.us/submit-manuscript.php
9. Burkle CM, Harrison BA, Koenig LF, Decker PA, Warner DO, et al. (2004) Morbidity and mortality of deep sedation in outpatient bone marrow biopsy. Am J Hematol 77(3): 250-256.

10. Islam A (2007) Bone marrow aspiration before bone marrow biopsy using the same bone marrow biopsy needle: A good or bad practice? J Clin Pathol 60(2): 212-215.

11. Drayson M (2003) Assessing the Myeloma cell load. RC Path: Education in Pathology 124: 19-20.

12. Islam A (2018) Bone Marrow Solid Core Biopsy Needle: A Critical Assessment of the Utility, Benefits and Limitations of the Instruments Employed in Current Day Haematology and Oncology. J Clin Pathol 71(6): 475-482.

13. Barnett TT, Reynolds JM, Shakespeare A, Dye P, Rappaport ES, et al (2014) A Prospective Comparison of Bone Marrow Biopsy Adequacy with and without Use of Powered Assistive Drill in the Clinical Setting Versus Interventional Radiology. Blood 124: 4823.

14. Swords RT, Kelly KR, Cohen SC, Miller LJ, Philbeck TE, et al. (2010) Rotary powered device for bone marrow aspiration and biopsy yields excellent specimens quickly and efficiently. J Clin Pathol 63(6): 562-565.

15. Kenneth E Symington, Felix Martinez Jr, Stephen C Cohen, Larry J Miller, Thomas E Philbeck (2013) CT-Guided Biopsy Produces High Quality Specimens For Diagnosis: A Retrospective Analysis Of 64 Consecutive Cases Using a Powered Biopsy Device At a Community Based Hospital. Blood 122: 5381

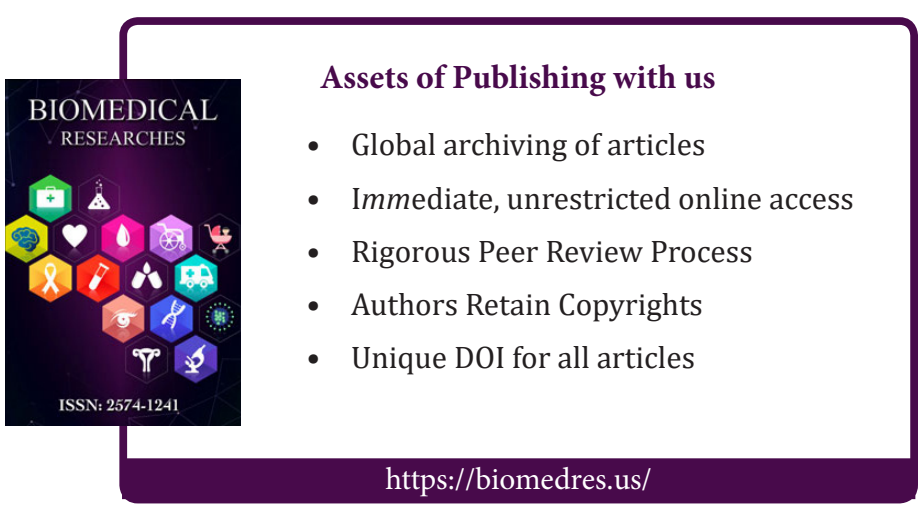

